\title{
Development of a Multicommutated Flow System with Chemiluminometric Detection for Quantification of Gentamicin in Pharmaceuticals
}

\author{
Lúcia H. M. L. M. Santos, ${ }^{1}$ A. N. Araújo, ${ }^{1}$ Boaventura Reis, ${ }^{2}$ and M. C. B. S. M. Montenegro ${ }^{1}$ \\ ${ }^{1}$ REQUIMTE, Department of Chemistry, Faculty of Pharmacy, University of Porto, Rua Anibal Cunha 164, 4050-047 Porto, Portugal \\ ${ }^{2}$ Centro de Energia Nuclear na Agricultura, Universidade de São Paulo, P.O. Box 96, 13400-970 Piracicaba, Brazil
}

Correspondence should be addressed to M. C. B. S. M. Montenegro, mcbranco@ff.up.pt

Received 13 July 2010; Accepted 2 September 2010

Academic Editor: F. R. P. Rocha

Copyright (c) 2010 Lúcia H. M. L. M. Santos et al. This is an open access article distributed under the Creative Commons Attribution License, which permits unrestricted use, distribution, and reproduction in any medium, provided the original work is properly cited.

\begin{abstract}
A new flow procedure based on multicommutation with chemiluminometric detection was developed to quantify gentamicin sulphate in pharmaceutical formulations. This approach is based on gentamicin's ability to inhibit the chemiluminometric reaction between luminol and hypochlorite in alkaline medium, causing a decrease in the analytical signal. The inhibition of the analytical signal is proportional to the concentration of gentamicin sulphate, within a linear range of 1 to $4 \mu \mathrm{g} \mathrm{mL}^{-1}$ with a coefficient variation $<3 \%$. A sample throughput of 55 samples $\mathrm{h}^{-1}$ was obtained. The developed method is sensitive, simple, with low reagent consumption, reproducible, and inexpensive, and when applied to the analysis of pharmaceutical formulations (eye drops and injections) it gave results with RSD between 1.10 and $4.40 \%$.
\end{abstract}

\section{Introduction}

Gentamicin is an aminoglycoside antibiotic with a wide broad spectrum activity. It is produced by Micromonospora purpurea and $M$. echinospora as a mixture of five major related components designated as gentamicin $C_{1}, C_{1 \mathrm{a}}, C_{2}$, $C_{2 \mathrm{a}}$, and $C_{2 \mathrm{~b}}$. These compounds are pseudotrisaccharides containing a garasone and a purpurosamine glycosidically linked to 2-deoxystreptamine, which differs from each one in their degree of methylation on the purpurosamine ring [1-3].

Gentamicin has been quantified in different matrices such as biological samples [4-6], pharmaceutical formulations [7-9], foodstuffs [10-12], and environmental samples $[13,14]$. Most of the proposed methods are based on separation techniques $[4-7,11-14]$ with different detection systems, conventional fluorimetric methodologies [8, 9], or immunoassays [10]. Due to lack of chromophores and fluorophores groups in its structure, it is difficult to quantify directly the gentamicin by using photometric or fluorimetric methods. Although the procedures are very sensitive, they are also expensive, because most of them rely on sophisticated methods and are often difficult to operate $[4,11,12,14]$. In addition, most of the time the sample needs to be prederivatized, which is a time-consuming process and involves toxic and environmentally unfriendly reagents $[5,6]$.

The official methods for the assay of gentamicin sulphate described in different Pharmacopoeias are microbiological for pharmaceutical preparations [15-17] and for raw materials is pointed out an HPLC method with UV detection for identification of the product [18]. The biological methods [15-17] described for pharmaceutical preparations are time consuming, of difficult operation, and with low detectability and precision as well as being subject to random error due to the inherent variability of biological responses. For such samples, in particular, other alternative methods have been presented in literature. Most of them are based mainly on chromatographic separation techniques with UV detection $[19,20]$, pulsed electrochemical detection $[21,22]$, and evaporative light-scattering detection (ELSD) [7, 23]. Fluorimetry $[8,9]$, indirect spectrophotometry $[24,25]$, potentiometry [26], and amperometry [27] methods are also described. 
Chemiluminescence (CL) can be defined as the production of electromagnetic radiation (ultraviolet, visible, or infrared) as a consequence of a chemical or biochemical reaction. CL has become a powerful tool for drug determination. It has wide-range applications, and low detection limits can be measured with a simple, low-cost instrument $[28,29]$. The association of CL with techniques based on continuous flow systems has allowed to develop highly sensitive analytical methods, which improves the accuracy of measurements, increases the sample throughput, and reduces considerably the reagent consumption and consequently the production of waste when compared with conventional procedures [30]. With continuous flow systems it is also possible to prepare online unstable reagents in a more efficient way [31]. In the literature two flow-injection analysis systems (FIA) applying sensitized chemiluminescence detection have been described for gentamicin $[31,32]$. In the first method, it is necessary to electrogenerate the oxidant reagent $(\mathrm{Co}(\mathrm{III}))$ that is unstable [31], while in the second method, gentamicin has to be previously derivatized to be able to participate in the chemiluminometric reaction [32].

Among the various techniques of continuous flow, multicommutation flow-injection analysis (MCFIA) is an alternative to increase versatility of flow systems. The flow system manifold comprised a set of solenoid valves assembled to work as independent commutation units, which controlled by microcomputer allows facilities to insert sample and reagent solutions following an intermittent pattern [33]. This process has the advantage of reducing both reagent consumption and generation of waste. Sample and reagent solutions can be handled using a single pumping channel, thus simplifying the manifold [34].

In this paper, an analytical method for the determination of gentamicin sulphate in pharmaceutical formulations employing a multicommutated flow system coupled with a chemiluminometric detector was developed. The detection process is based on a simple reaction, using commonand low-toxic reagents. In this case, for the first time, the reaction between luminol and hypochlorite, in alkaline medium, is used to quantify gentamicin, due to its capacity to inhibit the analytical signal. The usefulness of the proposed method was tested with commercial pharmaceutical formulations.

\section{Experimental}

2.1. Chemicals. Gentamicin sulphate was purchased from Sigma-Aldrich (St Louis, MO, USA); luminol, sodium hypochlorite, and sodium carbonate decahydrate from Fluka (Buchs, Switzerland); sodium hydrogen carbonate and hydrochloric acid from Merck (Darmstadt, Germany); sodium hydroxide from Riedel-de Haën (Seelze, Germany). All reagents were of analytical grade and all solutions were prepared with Milli-Q water (conductivity $<0.1 \mu \mathrm{S} \mathrm{cm}^{-1}$ ) recently boiled.

The pharmaceutical formulations (eye drops and injections) were obtained in the local market.
2.2. Preparation of Solutions. A $5.0 \times 10^{-3} \mathrm{~mol} \mathrm{~L}^{-1}$ luminol stock solution was prepared by dissolving $22.2 \mathrm{mg}$ of 5-amino-2,3-dihydro-1,4-phthalazinedione in $25 \mathrm{~mL}$ of $0.1 \mathrm{~mol} \mathrm{~L}^{-1}$ sodium carbonate solution, with $\mathrm{pH}$ adjusted to 9.2. A working solution of $2.0 \times 10^{-4} \mathrm{~mol} \mathrm{~L}^{-1}$ was daily prepared by dilution of the stock solution in the same buffer.

A $2.0 \times 10^{-5} \mathrm{~mol} \mathrm{~L}^{-1}$ sodium hypochlorite solution was also daily prepared by dilution in carbonate buffer $\mathrm{pH} 9.5$ of a sodium hypochlorite solution obtained from Fluka. Every week, the concentration of the commercial sodium hypochlorite solution was checked. For that a $1 \%(\mathrm{v} / \mathrm{v})$ solution of sodium hypochlorite was prepared from the commercial solution. The $\mathrm{pH}$ was adjusted to 6.2 with diluted sulfuric acid, and the concentration was determined spectrophotometrically by measuring its absorbance at 235 $\mathrm{nm}$ and applying a molar absorptivity of $100 \mathrm{~L} \mathrm{~mol}^{-1} \mathrm{~cm}^{-1}$ [35].

A $100 \mu \mathrm{g} \mathrm{mL}^{-1}$ stock solution of gentamicin was daily prepared by dissolving $5.0 \mathrm{mg}$ of gentamicin sulphate in $50 \mathrm{~mL}$ of water. Working aqueous standard solutions ranging from 1 to $4 \mu \mathrm{g} \mathrm{mL}^{-1}$ were prepared by dilution from the latter.

\subsection{Analysis of Commercial Pharmaceutical Formulations.} For eye drops formulations, a simple dilution with water was performed prior analysis in order to get a final gentamicin concentration within the linear range of the method (1$4 \mu \mathrm{g} \mathrm{mL}^{-1}$ ). A similar approach was used for injectable drugs. However, because of the high content of sodium bisulphite in their composition, added as preservative, it was necessary to carry out a procedure adapted from Amorim et al. [36]. A volume corresponding to $0.5 \mathrm{~mL}$ of the injectable drug was diluted in $14.5 \mathrm{~mL}$ of water and acidified with $50 \mu \mathrm{L}$ of concentrated hydrochloric acid. Afterwards, the solution was bubbled with nitrogen for 20 minutes to release the dissolved sulfur dioxide and neutralized with sodium hydroxide. Finally a dilution with water was carried out in order to obtain a gentamicin content included in the analytical range of the method.

2.4. Apparatus. Measurements were performed with a chemiluminescence detector comprising a Hamamatsu HS578404 (Hertfordshire, UK) photosensor module assembled with a spiral-shaped flow cell ( $40 \mu \mathrm{L}$ internal volume) constructed in Perspex, which was attached to the photosensor module window [37]. The multicommutation flow system depicted in Figure 1(a) comprises three three-way solenoid valves 161 T031 (NResearch Inc., West Caldwell, USA) and a Minipuls 3 peristaltic pump (Gilson, Viliers-le-Bell, France) with PVC pumping tubes (i.d. $=0.76$ and $1.3 \mathrm{~mm}$ ) as the propulsion system. The different components of the multicommutated flow system were connected with $0.8 \mathrm{~mm}$ i.d. PTFE tubing and a reaction coil of $50 \mathrm{~cm}$ was made with the same PTFE tube. Homemade confluence connectors were also used. Control of the analytical system, data acquisition and processing were carried out by means of a microcomputer coupled to a PC-LABCard model PCL711B Advantech (American Advantech Corp., San Jose, CA) 


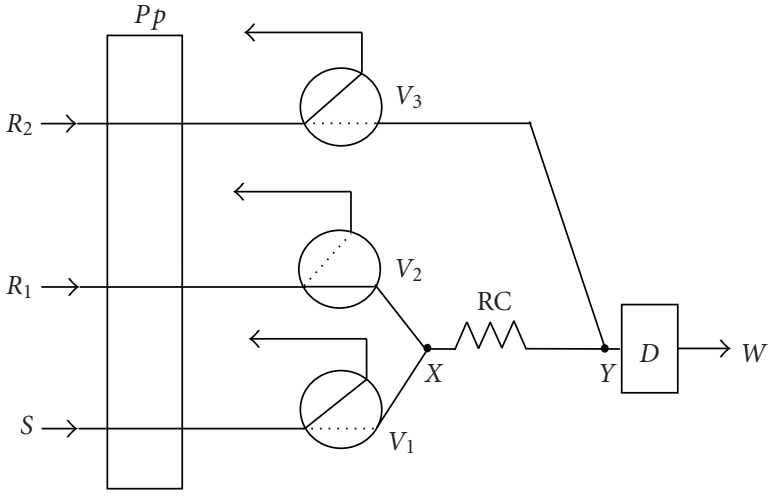

(a)

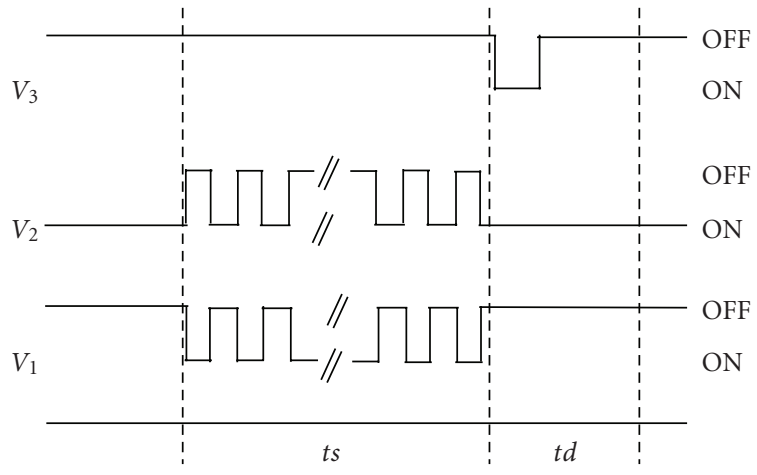

(b)

Figure 1: (a) scheme of the multicommutated flow system employed. $S$ : sample; $R_{1}$ : hypochlorite solution (carrier solution); $R_{2}$ : luminol solution; $P p$ : peristaltic pump; $V_{1}, V_{2}$, and $V_{3}$ : solenoid valves; $X$ and $Y$ : confluence points; RC: reaction coil; $D$ : chemiluminometric detector; $W$ : waste. (b) schematic representation of solenoid valves actuation during each analytical cycle. $V_{1}, V_{2}$, and $V_{3}$ : solenoid valves; $t s$ : sampling time; $t d$ : insertion time of luminol, reaction of this with the mixture sample/hypochlorite and signal detection.

electronic interface card and running a software written in QuickBasic Version 4.5.

Spectrophotometric measures for evaluating the concentration of commercial sodium hypochlorite solution were made by using a Perkin Elmer $45 \mathrm{UV} / \mathrm{Vis}$ double beam spectrophotometer and quartz cells with $1 \mathrm{~cm}$ of optical path.

2.5. Flow Injection Protocol. Three three-way solenoid valves $\left(V_{1}, V_{2}\right.$, and $\left.V_{3}\right)$ assessment to the system was enabled by the sample and reagents: $V_{1}$ for the sample $(S)$, while $V_{2}$ and $V_{3}$ inserted the hypochlorite $\left(R_{1}\right)$ and luminol $\left(R_{2}\right)$ solutions, respectively. The hypochlorite solution worked also as the carrier solution.

The analytical cycle (Figure 1(b)) started with the binary sampling of sample and hypochlorite solutions into the analytical path by the alternate actuation of solenoid valves $V_{1}$ and $V_{2}$, during a fixed time interval. Afterwards, valve $V_{1}$ was switched off, while valve $V_{2}$ had remained switched on, thus hypochlorite flowed continuously to displace the mixture sample/hypochlorite towards the confluence $Y$, where luminol solution was added through the solenoid valve $V_{3}$. The signal acquisition started with the introduction of luminol solution and persisted for a pre-defined period of time, after the valve $V_{3}$ has been switched OFF. Sample concentration was evaluated by the decrease of the monitored analytical signal, corresponding to the hypochlorite consumption by gentamicin. In the proposed procedure, the analytical signal related to the blank solution (reference signal) corresponded to the maximum of chemiluminescence emission.

\subsection{Experimental Procedures}

2.6.1. Optimized Flow System and Chemical Conditions. The quantification of gentamicin was performed by using a $2.0 \times$ $10^{-4} \mathrm{~mol} \mathrm{~L}^{-1}$ luminol solution with a $\mathrm{pH}$ of 9.2 and a $2.0 \times$ $10^{-5} \mathrm{~mol} \mathrm{~L}^{-1}$ hypochlorite solution with a $\mathrm{pH}$ of 9.5 , at room temperature. On each analysis were used $725 \mu \mathrm{L}$ and $41.7 \mu \mathrm{L}$ of sample and luminol volumes, respectively. The flow rate of sample and hypochlorite was $2.9 \mathrm{~mL} \mathrm{~min}^{-1}$ and the luminol one was $1.15 \mathrm{~mL} \mathrm{~min}^{-1}$.

2.6.2. Calibration Curve Using Gentamicin Sulphate. A series of working standards solutions of gentamicin sulphate, prepared in water, in the range of 1 to $4 \mu \mathrm{g} \mathrm{mL}^{-1}$, were measured in triplicate and the difference of signal relatively to the blank was used to construct the corresponding calibration curve.

2.6.3. Recovery Studies. Recoveries studies were made by using the proposed method, after addition of $0.6-1.0 \mathrm{~mL}$ of a $100 \mu \mathrm{g} \mathrm{mL}{ }^{-1}$ gentamicin sulphate solution to $100 \mathrm{~mL}$ of sample solution, in order to achieve a gentamicin concentration around of $2.0 \mu \mathrm{g} \mathrm{mL}^{-1}$, which is in the linear range of the developed method.

\section{Results and Discussion}

The proposed method is based on the ability of gentamicin to inhibit the oxidation reaction between luminol and hypochlorite in alkaline medium. This aminoglycoside antibiotic has several amine groups prone to oxidation by hypochlorite [38-41], resulting in an inhibition of the analytical signal, which is proportional to the concentration of gentamicin. Since the method is based on the chemiluminometric signal inhibition, a maximization of the reference signal is necessary in order to improve the analytical signal.

3.1. Optimisation of Chemical Conditions. In order to achieve the maximum of sensitivity of the reaction some chemical variables were studied such as the influence of concentration and $\mathrm{pH}$ of hypochlorite and luminol solutions involved in the reaction by using a univariate approach. Hypochlorite concentrations were investigated from $5.0 \times 10^{-6}$ to 3.5 $\times 10^{-5} \mathrm{~mol} \mathrm{~L}^{-1}$, and the results showed that inhibition of chemiluminescence signal was higher for the concentration of $2.0 \times 10^{-5} \mathrm{~mol} \mathrm{~L}^{-1}$. The influence of $\mathrm{pH}$ of hypochlorite solution in the reaction was studied in the range from 9 to 


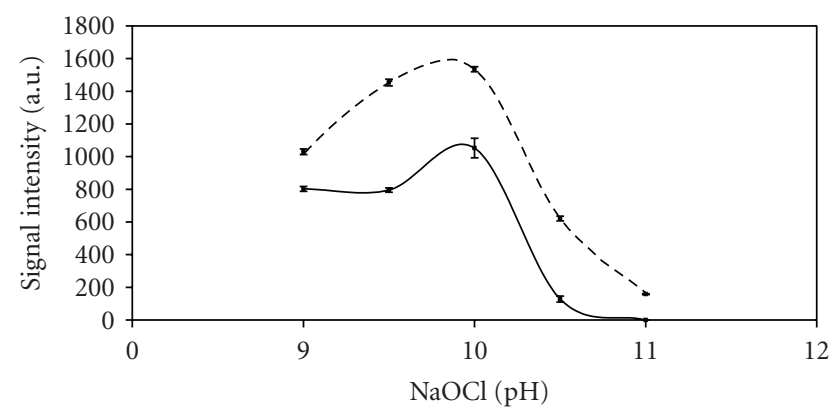

FIGURE 2: Influence of hypochlorite's $\mathrm{pH}$ in the analytical signal. Dotted line: Reference signal; Continue line: Gentamicin standard solution $\left(1 \mu \mathrm{g} \mathrm{mL}^{-1}\right)$.

11 (Figure 2). The reference signal intensity was higher for $\mathrm{pH} 10$, although the inhibition effect of gentamicin was more pronounced for $\mathrm{pH}$ 9.5. Hence this $\mathrm{pH}$ value was chosen for further studies.

A similar study varying the concentration of luminol solution between $5.0 \times 10^{-5}$ and $4.0 \times 10^{-4} \mathrm{~mol} \mathrm{~L}^{-1}$ was performed, and a higher decrease of the chemiluminescence signal was observed for a luminol concentration of 2.0 $\times 10^{-4} \mathrm{~mol} \mathrm{~L}^{-1}$. The influence of the $\mathrm{pH}$ in the luminol reaction was investigated using buffer solutions in a $\mathrm{pH}$ range from 8 to 12.6. The analytical signal increased with the increase of the $\mathrm{pH}$ of luminol solution. However, at a $\mathrm{pH}$ value of 9.5 occurred a saturation of the detector, whereby it was decided to use a luminol solution with $\mathrm{pH}$ adjusted to 9.2, as a compromise between the analytical signal intensity, the hypochlorite concentration previously selected, and the detector saturation.

3.2. Optimisation of Flow System Conditions. To obtain a good correlation between the inhibition of the analytical signal and the concentration of gentamicin, flow parameters such as the time of reaction between hypochlorite and gentamicin, the volume of sample inserted, and the flow rate were also studied.

The time of reaction between hypochlorite and gentamicin is important to obtain a maximum rate in the signal inhibition. In order to achieve this purpose, reaction coils with lengths of $12.8,50$, and $100 \mathrm{~cm}$ were inserted between the two confluences $X$ and $Y$ as shown in Figure 1(a). The results reveal that a higher inhibition of the analytical signal was obtained for a reaction coil of $50 \mathrm{~cm}$.

The influence of the sample volume was also investigated varying the time interval for its insertion between 9.0 to 30.0 seconds, corresponding to sample volumes from 435 to $1450 \mu \mathrm{L}$, respectively. A higher inhibition of the analytical signal was achieved for $725 \mu \mathrm{L}$ of sample solution, which corresponds to 10 sampling cycles with a time interval of $1.5 \mathrm{~s}$.

The flow rate is another critical parameter for the chemiluminescence detection because it defines the residence time of the sample zone in the flow cell. Flow rates between 2.1 and $3.5 \mathrm{~mL} \mathrm{~min}^{-1}$ for sample and hypochlorite solutions as well as from 0.85 to $1.45 \mathrm{~mL} \mathrm{~min}^{-1}$ for luminol solution
TABLE 1: Comparision of different chemiluminometric methodologies applied to flow systems for determination of gentamicin in pharmaceutical formulations.

\begin{tabular}{lccc}
\hline & Li et al. [31] & $\begin{array}{c}\text { Ramos-Fernández } \\
\text { et al. [32] }\end{array}$ & $\begin{array}{c}\text { Proposed } \\
\text { method }\end{array}$ \\
\hline $\begin{array}{l}\text { Sample volume } \\
(\mu \mathrm{L})\end{array}$ & 100 & 1000 & 725 \\
$\begin{array}{l}\text { Gentamicin } \\
\text { prederivatization } \\
\text { Linear range } \\
\left(\mu \mathrm{mL}^{-1}\right)\end{array}$ & No & Yes (with OPA) & No \\
$\begin{array}{l}\text { Detection limit } \\
\left(\mu \mathrm{g} \mathrm{mL}^{-1}\right)\end{array}$ & $0.01-80$ & $3.93-30$ & $1-4$ \\
$\begin{array}{l}\text { Sample } \\
\text { throughput }\end{array}$ & $\sim 60 \mathrm{~h}^{-1}$ & Not described & $55 \mathrm{~h}^{-1}$ \\
$\begin{array}{l}\text { Intermediate } \\
\text { precision }(\%)\end{array}$ & $<3.2$ & Not described & $<3$ \\
\hline
\end{tabular}

OPA: $o$-phthalaldehyde.

were tested. It was noticed that the reference range of signal intensity was higher when the flow rate was $3.5 \mathrm{~mL} \mathrm{~min}^{-1}$ for the sample and hypochlorite solutions conjugated with $1.45 \mathrm{~mL} \mathrm{~min}^{-1}$ for luminol. However, the inhibition effect of gentamicin was more pronounced for a flow rate of $2.9 \mathrm{~mL} \mathrm{~min}^{-1}$ (sample and hypochlorite solutions) and $1.15 \mathrm{~mL} \mathrm{~min}^{-1}$ (luminol solution). Therefore, these flow rates were chosen for the following studies.

3.3. Interferences. The interference of chemical compounds such as arginine, sodium bisulphite, cetrimide, benzalkonium chloride, sodium chloride, potassium dihydrogenphosphate, EDTA, monosodium phosphate, disodium phosphate, glycerol, methylparaben, propylparaben, hydroxyl$\beta$-cyclodextrin, sodium mercuriothiolate, PVP, dexamethasone, indometacin, and tetryzoline, usually used as excipients in pharmaceutical formulations (eye drops and injections), was studied by using a $2.5 \times 10^{-6} \mathrm{~mol} \mathrm{~L}^{-1}$ of fixed concentration of gentamicin. This solution was spiked with the referred substances, at different proportions. Proportions of interfering/gentamicin of $100: 1,50: 1,25: 1,10: 1$, and 1:1 were tested. A chemical compound would be considered to interfere if its presence resulted in a variation of the analytical signal around $3 \%( \pm 3 \%)$ relative to the analytical signal obtained without excipient [37]. The results showed a variation of analytical signal higher then 19\% for $1: 1$ proportion for arginine, sodium bisulphite, and sodium mercuriothiolate species. Nevertheless, arginine and sodium mercuriothiolate are presented in pharmaceutical formulations but at very low concentrations when compared with those tested. The probability of interfering with the analytical determination is low, as evidenced by tests carried out in Indobiotic eye drops formulation. However, for the sodium bisulphite, there was a variation of the analytical signal of around $20 \%$ even for the lowest proportions of interfering. Therefore, in these cases, after acidification, samples were bubbled with a nitrogen stream. The nitrogen bubbling time was evaluated for a period of time between 
TABLE 2: Determination of gentamicin sulphate in different pharmaceutical formulations.

\begin{tabular}{lccc}
\hline Pharmaceutical formulation & $\begin{array}{c}\text { Concentration } \\
\text { declared }\left(\mathrm{mg} \mathrm{mL}^{-1}\right)\end{array}$ & $\begin{array}{c}\text { Concentration } \\
\text { found }\left(\mathrm{mg} \mathrm{mL}^{-1}\right)^{\mathrm{a}}\end{array}$ & \\
\hline Eye drops & 4.42 & $4.41 \pm 0.15$ & 3.33 \\
$\quad$ Gentocil & 3.00 & $2.99 \pm 0.13$ & 4.32 \\
Ophtagram & 5.08 & $5.13 \pm 0.16$ & 3.11 \\
Indobiotic & 5.00 & $4.90 \pm 0.05$ & 1.10 \\
Dexamytrex Ophtiole & 4.42 & $4.44 \pm 0.06$ & \\
Colircusí Gentadexa & & $81 \pm 4$ & \\
Injection & 80 & & \\
$\quad$ Garalone &
\end{tabular}

${ }^{a}$ Each determination corresponds to an average \pm standard deviation of three analyses made with two samples from the same package.

10 and 30 minutes. It was observed that a time of 20 minutes was enough to reduce the analytical signal to values near the corresponding signal of gentamicin for the same proportion.

3.4. Figures of Merit for the Proposed Method. After optimisation of the flow system relative to chemical and flow conditions, it was possible to achieve an analytical linear range between 1 and $4 \mu \mathrm{g} \mathrm{mL}^{-1}$ of gentamicin and detection limit of $0.023 \mu \mathrm{g} \mathrm{mL}^{-1}$. For a confidence degree of $95 \%$, the calibration curve is expressed as $\Delta E=210( \pm 7) C+6$ $( \pm 16), \Delta E$ being the variation of the analytical signal, in arbitrary units, and $C$ being the concentration of gentamicin sulphate, in $\mu \mathrm{g} \mathrm{mL}^{-1}$. A coefficient of variation between 0.58 and $2.34 \%(n=10)$ was obtained, for the analysis of standard solutions of gentamicin within the analytical linear range of the proposed method. RSD values lower than $3 \%$ were acquired for the intermediate precision. The proposed method allowed a sample throughput of 55 samples $\mathrm{h}^{-1}$.

Table 1 compares the results obtained with those given by other authors, for the assay of gentamicin in flow conditions $[31,32]$ proved that the limit of detection were of the same order of magnitude. The accuracy of the results was, however, better than those given in [31] and also was the sampling rate $[31,32]$.

3.5. Analysis of Pharmaceutical Formulations. The proposed method was applied to the determination of gentamicin sulphate in commercial pharmaceutical formulations (eye drops and injections) commercialized in Portugal. The results obtained are summarized in Table 2 and are in good agreement with the labelled content. In order to evaluate the validity of the proposed method for the determination of gentamicin in pharmaceutical formulations, recovery studies were carried out on samples to which known amounts of gentamicin were added. A mean of recovery value of about $99.5 \pm 3.3 \%$ was obtained.

The officially accepted methods for the determination of gentamicin in pharmaceutical preparations are based on microbiological assays [15-17]. The biological variability of results obtained does not allow to correlate with the analytical results obtained by the proposed method. In order to validate the analytical results, we tried to adapt a method based on HPLC with UV detection, proposed by the British Pharmacopeia and used for identification of raw materials [18]. In this, the proportion of main components of gentamicin (gentamicin $C_{1}, C_{1 \mathrm{a}}, C_{2}$, and $C_{2 \mathrm{a}}$ ) is assessed after its derivatization with $o$-phthalaldehyde and mercaptoacetic acid. When trying to adapt this method to the analysis of pharmaceutical products, the proportion of the main components of gentamicin in the standards was not equivalent to the real samples, so it was not possible to correlate the total areas of peaks with the concentration of gentamicin. This difference was observed for all the samples.

\section{Conclusions}

The described multicommutated flow system with chemiluminometric detection allowed an exact and rapid quantification of gentamicin sulphate in pharmaceutical formulations (eye drops and injections). Besides being economic, the proposed flow system has the advantage of producing a low waste of residues when compared with other methods based on flow techniques, previously proposed [31, 32], being versatile enough and easy to operate. The chemiluminescence reaction proposed for detection uses common- and low-toxic reagents, contrary to the methods proposed by other authors $[31,32]$, which makes the method more environmentally friendly.

This approach represents an advantageous alternative to the officially accepted microbiological method, which can be implemented in a simple manner in any laboratory quality control in pharmaceutical industry.

\section{Acknowledgment}

L. H. M. L. M. Santos thanks FCT for the PhD Grant (SFRH/BD/48168/2008).

\section{References}

[1] D. J. Cooper, M. D. Yudis, R. D. Guthrie, and A. M. Prior, "The gentamicin antibiotics. Part I. Structure and absolute stereochemistry of methyl garosaminide," Journal of the Chemical Society C, pp. 960-963, 1971. 
[2] D. J. Cooper, M. D. Yudis, H. M. Marigliano, and T. Traubel, "The gentamicin antibiotics. II. Separation and degradation of the gentamicin $\mathrm{C}$ components. The purpurosamines, a new class of naturally occurring 2,6-diaminomonosaccharides," Journal of the Chemical Society C, vol. 17, pp. 2876-2879, 1971.

[3] D. J. Copper, P. J. L. Daniels, M. D. Yudis, H. M. Marigliano, R. D. Guthrie, and S. T. Bukhari, "The gentamicin antibiotics. 3. The gross structures of the gentamicin C components," Journal of the Chemical Society C, vol. 18, pp. 3126-3129, 1971.

[4] C. Lecároz, M. A. Campanero, C. Gamazo, and M. J. Blanco-Prieto, "Determination of gentamicin in different matrices by a new sensitive high-performance liquid chromatography-mass spectrometric method," Journal of Antimicrobial Chemotherapy, vol. 58, no. 3, pp. 557-563, 2006.

[5] A. I. Al-Amoud, B. J. Clark, and H. Chrystyn, "Determination of gentamicin in urine samples after inhalation by reversedphase high-performance liquid chromatography using precolumn derivatisation with o-phthalaldehyde," Journal of Chromatography B, vol. 769, no. 1, pp. 89-95, 2002.

[6] E. Kaale, Y. Long, H. A. Fonge et al., "Gentamicin assay in human serum by solid-phase extraction and capillary electrophoresis," Electrophoresis, vol. 26, no. 3, pp. 640-647, 2005.

[7] N. C. Megoulas and M. A. Koupparis, "Development and validation of a novel LC/ELSD method for the quantitation of gentamicin sulfate components in pharmaceuticals," Journal of Pharmaceutical and Biomedical Analysis, vol. 36, no. 1, pp. 73-79, 2004.

[8] A. A. Al-Majed, F. Belal, M. A. Abounassif, and N. Y. Khalil, "Fluorimetric determination of gentamicin in dosage forms and biological fluids through derivatization with 4-chloro-7nitrobenzo-2-oxa-1,3-diazole (NBD-Cl)," Mikrochimica Acta, vol. 141, no. 1-2, pp. 1-6, 2003.

[9] M. Rizk, Y. El-Shabrawy, N. A. Zakhari, S. S. Toubar, and L. A. Carreira, "Fluorimetric determination of aminoglycoside antibiotics using lanthanide probe ion spectroscopy," Talanta, vol. 42, no. 12, pp. 1849-1856, 1995.

[10] R. M. Van Es, S. J. Setford, Y. J. Blankwater, and D. Meijer, "Detection of gentamicin in milk by immunoassay and flow injection analysis with electrochemical measurement," Analytica Chimica Acta, vol. 429, no. 1, pp. 37-47, 2001.

[11] D. N. Heller, S. B. Clark, and H. F. Righter, "Confirmation of gentamicin and neomycin in milk by weak cation-exchange extraction and electrospray ionization/ion trap tandem mass spectrometry," Journal of Mass Spectrometry, vol. 35, no. 1, pp. 39-49, 2000.

[12] W.-X. Zhu, J.-Z. Yang, W. Wei, Y.-F. Liu, and S.-S. Zhang, "Simultaneous determination of 13 aminoglycoside residues in foods of animal origin by liquid chromatographyelectrospray ionization tandem mass spectrometry with two consecutive solid-phase extraction steps," Journal of Chromatography A, vol. 1207, no. 1-2, pp. 29-37, 2008.

[13] J. M. Serrano and M. Silva, "Rapid and sensitive determination of aminoglycoside antibiotics in water samples using a strong cation-exchange chromatography non-derivatisation method with chemiluminescence detection," Journal of Chromatography A, vol. 1117, no. 2, pp. 176-183, 2006.

[14] D. Löffler and T. A. Ternes, "Analytical method for the determination of the aminoglycoside gentamicin in hospital wastewater via liquid chromatography-electrospray-tandem mass spectrometry," Journal of Chromatography A, vol. 1000, no. 1-2, pp. 583-588, 2003.

[15] European Pharmacopheia, 5th ed., Strasbourg, France, pp. 1653-1654, 2005.
[16] British Pharmacopeia, London, vol. III, pp. 2508-2509, 2005.

[17] The United States Pharmacopeia and The National Formulary, USP 23, NF 18, Rockville, Md, USA, pp. 895-896, 2005.

[18] British Pharmacopeia, London, vol. I, pp. 903-904, 2005.

[19] E. Kaale, S. Leonard, A. Van Schepdael, E. Roets, and J. Hoogmartens, "Capillary Electrophoresis analysis of gentamicin sulphate with UV detection after pre-capillary derivatization with 1,2-phthalic dicarboxaldehyde and mercaptoacetic acid," Journal of Chromatography A, vol. 895, no. 1-2, pp. 67-79, 2000.

[20] B.-H. Kim, S. C. Lee, H. J. Lee, and J. H. Ok, "Reversedphase liquid chromatographic method for the analysis of aminoglycoside antibiotics using pre-column derivatization with phenylisocyanate," Biomedical Chromatography, vol. 17, no. 6, pp. 396-403, 2003.

[21] E. Adams, W. Roelants, R. De Paepe, E. Roets, and J. Hoogmartens, "Analysis of gentamicin by liquid chromatography with pulsed electrochemical detection," Journal of Pharmaceutical and Biomedical Analysis, vol. 18, no. 4-5, pp. 689-698, 1998.

[22] V. Manyanga, K. Kreft, B. Divjak, J. Hoogmartens, and E. Adams, "Improved liquid chromatographic method with pulsed electrochemical detection for the analysis of gentamicin," Journal of Chromatography A, vol. 1189, no. 1-2, pp. 347354, 2008.

[23] I. Clarot, P. Chaimbault, F. Hasdenteufel, P. Netter, and A. Nicolas, "Determination of gentamicin sulfate and related compounds by high-performance liquid chromatography with evaporative light scattering detection," Journal of Chromatography A, vol. 1031, no. 1-2, pp. 281-287, 2004.

[24] A. M. El-Didamony, A. S. Amin, A. K. Ghoneim, and A. M. Telebany, "Indirect spectrophotometric determination of gentamicin and vancomycin antibiotics based on their oxidation by potassium permanganate," Central European Journal of Chemistry, vol. 4, no. 4, pp. 708-722, 2006.

[25] J. Krzek, H. Woltyńska, and U. Hubicka, "Determination of gentamicin sulphate in injection solutions by derivative spectrophotometry," Analytical Letters, vol. 42, no. 3, pp. 473482, 2009.

[26] E. G. Kulapina, V. V. Baraguzina, and O. I. Kulapina, "Rapid potentiometric determination of aminoglycoside antibiotics in drug dosage forms and biological fluids using ion-selective electrodes," Journal of Analytical Chemistry, vol. 60, no. 6, pp. 592-597, 2005.

[27] E. V. Khaldeeva, E. P. Medyantseva, N. A. Imanaeva, and G. K. Budnikov, "Determination of gentamicin with an amperometric enzyme immunosensor," Journal of Analytical Chemistry, vol. 57, no. 12, pp. 1097-1102, 2002.

[28] A. C. Calokerinos, N. T. Deftereos, and W. R. G. Baeyens, "Chemiluminescence in drug assay," Journal of Pharmaceutical and Biomedical Analysis, vol. 13, no. 9, pp. 1063-1071, 1995.

[29] Y. F. Mestre, L. L. Lahuerta Zamora, and J. M. Calatayud, "Flow-chemiluminescence: a growing modality of pharmaceutical analysis," Luminescence, vol. 16, no. 3, pp. 213-235, 2001.

[30] K. Mervartová, M. Polášek, and J. Martínez Calatayud, "Recent applications of flow-injection and sequential-injection analysis techniques to chemiluminescence determination of pharmaceuticals," Journal of Pharmaceutical and Biomedical Analysis, vol. 45, no. 3, pp. 367-381, 2007.

[31] B. Li, Z. Zhang, and M. Wu, "Flow injection chemiluminescence determination of gentamycin using on- line electrogenerated $\mathrm{Co}(\mathrm{III})$ as the oxidant," Analytical Letters, vol. 33, no. 8, pp. 1577-1590, 2000. 
[32] J. M. Ramos-Fernández, A. M. García-Campaña, F. AlésBarrero, and J. M. Bosque-Sendra, "Determination of gentamicin in pharmaceutical formulations using peroxyoxalate chemiluminescent detection in flow-injection analysis," Talanta, vol. 69, no. 3, pp. 763-768, 2006.

[33] B. F. Reis, M. F. Giné, E. A. G. Zagatto, J. L. F. C. Lima, and R. A. Lapa, "Multicommutation in flow analysis. Part 1. Binary sampling: concepts, instrumentation and spectrophotometric determination of iron in plant digests," Analytica Chimica Acta, vol. 293, no. 1-2, pp. 129-138, 1994.

[34] F. R. P. Rocha, B. F. Reis, E. A. G. Zagatto, J. L. F. C. Lima, R. A. S. Lapa, and J. L. M. Santos, "Multicommutation in flow analysis: concepts, applications and trends," Analytica Chimica Acta, vol. 468, no. 1, pp. 119-131, 2002.

[35] L. M. Magalhães, M. A. Segundo, S. Reis, J. L. F. C. Lima, J. M. Estela, and V. Cerdà, "Automatic in vitro determination of hypochlorous acid scavenging capacity exploiting multisyringe flow injection analysis and chemiluminescence," Analytical Chemistry, vol. 79, no. 10, pp. 3933-3939, 2007.

[36] C. G. Amorim, A. N. Araújo, and M. C. B. S. M. Montenegro, "Exploiting sequential injection analysis with lab-on-valve and miniaturized potentiometric detection. Epinephrine determination in pharmaceutical products," Talanta, vol. 72, no. 4, pp. 1255-1260, 2007.

[37] J. A. V. Prior, J. L. M. Santos, and J. L.s F. C. Lima, "Automated chemiluminometric screening of counterfeit drugs of the antituberculosis agent pyrazinamide," Journal of AOAC International, vol. 92, no. 3, pp. 830-836, 2009.

[38] J. Li and P. K. Dasgupta, "Chemiluminescence detection with a liquid core waveguide: determination of ammonium with electrogenerated hypochlorite based on the luminolhypochlorite reaction," Analytica Chimica Acta, vol. 398, no. 1, pp. 33-39, 1999.

[39] C. Vannecke, E. Van Gyseghem, M. S. Bloomfield, T. Coomber, Y. V. Heyden, and D. L. Massart, "Development of a generic flow injection analysis method for compounds with a secondary amine or amide function, using an experimental design approach: I. Selection and evaluation of the FIA system parameters," Analytica Chimica Acta, vol. 446, no. 1-2, pp. 413-428, 2001.

[40] M. A. Sanchez and F. R. P. Rocha, "A flow-based analytical procedure for salbutamol determination exploiting chemiluminescence in a liquid-core waveguide," Analytical Letters, vol. 41, no. 9, pp. 1579-1591, 2008.

[41] G. G. Oliveira, B. C. Janegitz, M. B. Batistão, F. H. Salami, O. Fatibello-Filho, and O. D. Leite, "Determination of paracetamol using a flow injection analysis with multicommutation and chemiluminescence detection," Quimica Nova, vol. 32, no. 7, pp. 1755-1759, 2009. 


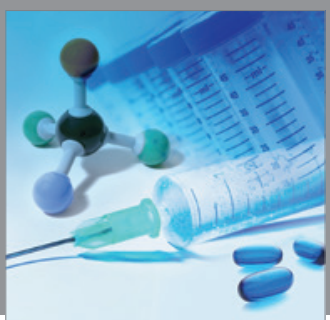

International Journal of

Medicinal Chemistry

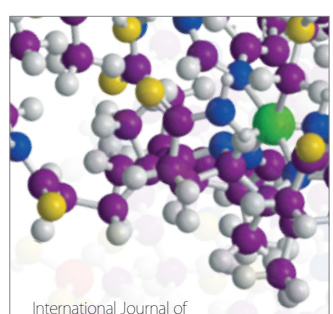

Carbohydrate Chemistry

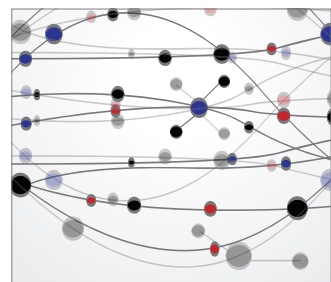

The Scientific World Journal
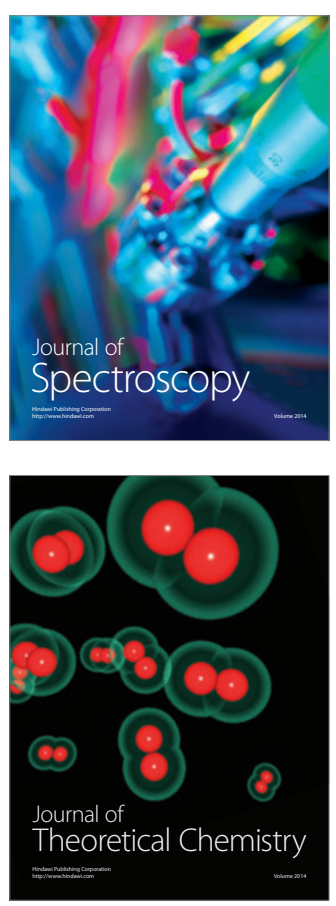
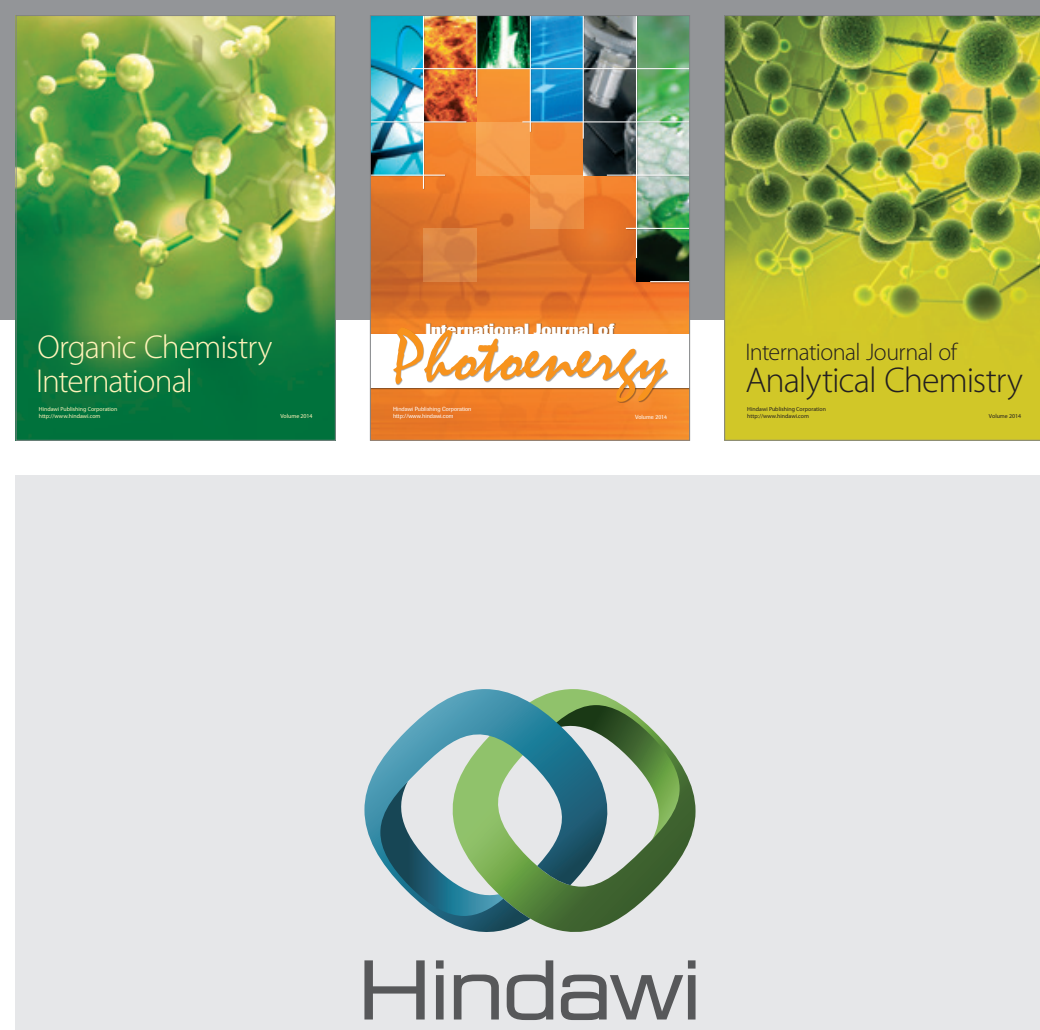

Submit your manuscripts at

http://www.hindawi.com
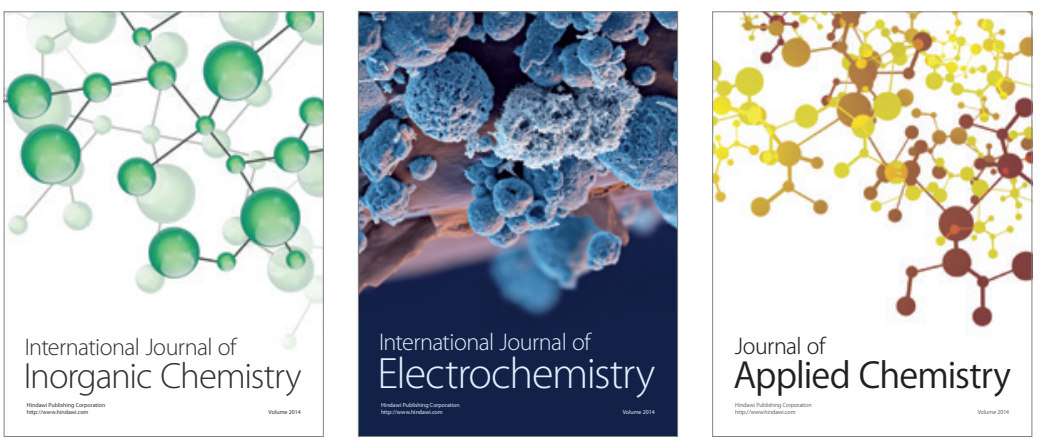

Journal of

Applied Chemistry
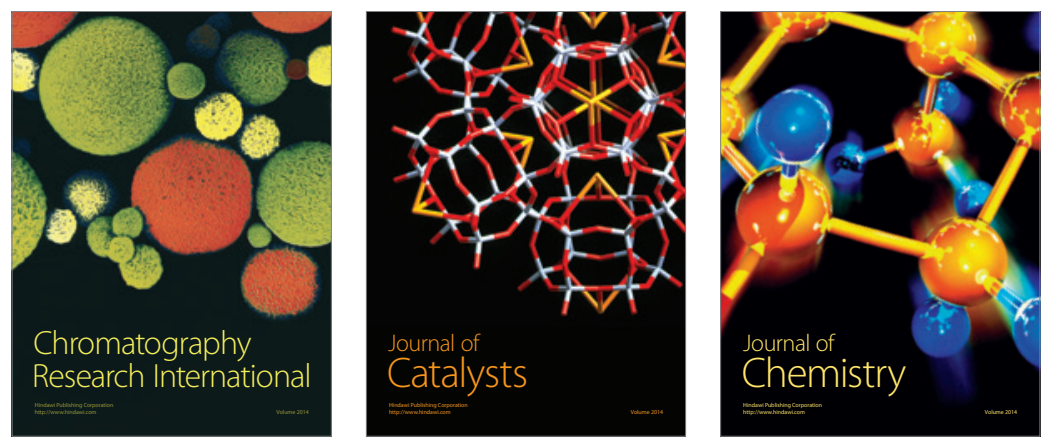
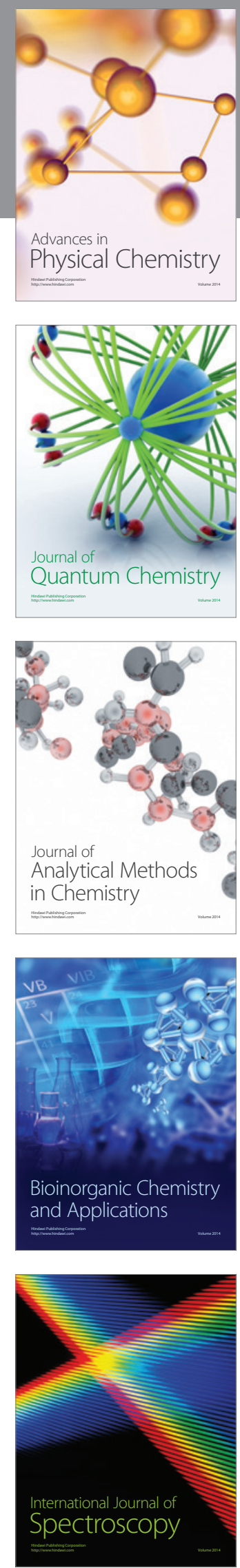